\section{What's hot that the other CLINIC lot got}

\author{
Elizabeth Batalla-Duran
}

\section{AZITHROMYCIN IN ASTHMA TREATMENT}

Azithromycin is being used in a variety of respiratory diseases for its anti-inflammatory effects as well as its antimicrobial properties. The AMAZES study (Gibson PG, et al. Lancet 2017;390:659-668) investigated whether azithromycin had any beneficial effects on asthma exacerbations and quality of life when added to standard therapy of inhaled corticosteroid and long-acting bronchodilators in patients with persistent symptomatic asthma. The randomised, double-blind placebo-controlled study allocated patients to receive azithromycin $500 \mathrm{mg}$ (217 patients) or placebo (207 patients) three times a week for 48 weeks. Primary endpoints were the number of severe or moderate exacerbations (measured by need for increased corticosteroids, hospital visit, antibiotics or a reduction in asthma control questionnaire score) and asthma quality of life. Secondary endpoints were asthma control, nasal symptoms, cough, respiratory infections and sputum production. Azithromycin significantly reduced overall asthma exacerbations $(1.07$ per patient-year $(95 \% \mathrm{CI} 0.85$ to 1.29$)$ compared with placebo (1.86 per patient-year (1.54 to 2.18)). The incidence rate ratio was $0.59(95 \% \mathrm{CI} 0.47$ to 0.74$)$. Azithromycin also significantly reduced severe exacerbations compared with placebo $(p=0.002)$. The proportion of patients experiencing at least one asthma exacerbation was reduced by azithromycin treatment $(94$ patients (44\%) compared with 127 (61\%) in the placebo group, $\mathrm{p}<0.0001)$. Azithromycin significantly improved asthma-related quality of life across all Asthma Quality of life questionnaire domains. The rate of antibiotic courses for respiratory infection was significantly reduced in the azithromycin treated group. Azithromycin was well tolerated with no significant difference in serious adverse events compared with the placebo group although there was significantly more diarrhoea in the azithromycin group. The authors note that there was an increase in

Correspondence to Dr Elizabeth Batalla-Duran,

Respiratory Medicine, Derriford Hospital, Plymouth PL6 8DH, UK; ebatalla-duran@nhs.net azithromycin-resistant organisms in bacterial cultures at the end of treatment which, although not statistically significant, could be a consideration for future study.

\section{PNEUMONIA ONLY ON CT JUST AS BAD AS ON X-RAY}

It is not uncommon for a patient to present with signs and symptoms of community acquired pneumonia (CAP) but have a normal chest X-ray (CXR) with subsequent CT showing evidence of consolidation. This American study (Upchurch CP, et al. Chest. doi:10.1016/j.chest.2017.07.035) sought to compare characteristics of CAP in patients with pneumonia on CXR with those with pneumonia on CT but not CXR. 2251 adults were studied (as part of a larger trial of pneumonia patients). 2185 (97\%) of these had pneumonia visible on CXR ('CXR pneumonia') while 66 (3\%) had pneumonia only visualised on CT performed within a day of a normal CXR ('CT only pneumonia'). Clinical characteristics, antibiotic use, pathogens identified and clinical outcomes were compared between the two groups.

Clinical profiles were similar between the two groups but those with CT-only pneumonia tended to be younger, more likely to present with chest pain and wheeze and have a higher BMI. Unsurprisingly, a smaller proportion of the CT-only pneumonia group received antibiotics within 6hours but by 24 hours results were similar. Antibiotic choice was similar across the two groups. Pathogen detection showed viruses were more common than bacteria in both groups but common bacterial CAP pathogens (Streptococcus pneumoniae, Mycoplasma pneumoniae and Staphylococcus aureus) were detected in similar proportions in the two groups. Clinical outcomes, including hospital length of stay, admission to intensive care, mechanical ventilation, shock and death were similar between CT-only pneumonia and CXR pneumonia. The authors therefore conclude patients presenting with CT-only pneumonia should be managed with the same principles and antibiotic regimen as CXR pneumonia. Given the similar outcomes and severity of morbidity, it is also important to consider a CT in patients with signs and symptoms suggestive of CAP with no CXR evidence of consolidation to ensure identification of pneumonia and guide appropriate management.

\section{AIR POLLUTION AND PULMONARY FIBROSIS}

Air pollution is implicated as an adverse factor in an increasing number of respiratory conditions. The latest to be put under the spotlight is idiopathic pulmonary fibrosis (IPF). It is thought a number of inhaled environmental toxins may cause an abnormal fibrotic response in the lungs, contributing to IPF. The authors of this study (Winterbottom CJ, et al. Chest. doi:10.1016/j.chest.2017.07.034) looked at the effect of PM10 and PM2.5 on the FVC of patients with IPF over time hypothesising that increased exposure to air pollution would cause a more rapid decline in lung function. 135 patients presenting to a single interstitial lung disease centre with definite or probable IPF were studied. Pollution data from air quality monitoring stations nearest to the subjects' address were available from the Environmental Protection Agency (USA) via an online tool. Mean daily concentrations of PM10 and PM2.5 were collected. Data were collated to come up with a mean air pollution exposure over time for each subject. Distance from a major road was also calculated to see if this had an effect on FVC. There was a significant relationship between increased exposure to PM10 and an accelerated FVC decline during the study period $(\mathrm{p}=0.019)$. Each $5 \mu \mathrm{g} / \mathrm{m}^{3}$ increase in average ambient PM10 concentration at a subjects' address corresponded with an additional $35 \mathrm{~mL} /$ year decline in FVC $(95 \% \mathrm{CI} 6$ to $65 \mathrm{~mL} /$ year). Exposure to PM2.5 did not show the same significant relationship. There was no significant effect on distance from a major road on the rate of FVC decline. The long-term effects of air pollution on respiratory health are becoming increasingly apparent. Perhaps more robust methods to reduce air pollution are needed.

\section{Competing interests None declared.}

Provenance and peer review Commissioned; internally peer reviewed.

(C) Article author(s) (or their employer(s) unless otherwise stated in the text of the article) 2017. All rights reserved. No commercial use is permitted unless otherwise expressly granted.

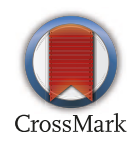

To cite Batalla-Duran E. Thorax 2017;72:964.

Thorax 2017;72:964.

doi:10.1136/thoraxjnl-2017-210964 